\title{
PERSPECTIVAS DAS AÇÕES INTERSETORIAIS, DE INCLUSÃO E PARTICIPAÇÃO SOCIAIS DESENVOLVIDAS POR AGENTES COMUNITÁRIOS DE SAÚDE
}

\author{
Perspectives of the intersectoral actions developed by the \\ community health workers towards social inclusion and \\ participation
}

\author{
Perspectivas de las acciones intersectoriales, de inclusión y \\ participación social desarrolladas por agentes comunitarios \\ de salud
}

Artigo Original

\begin{abstract}
RESUMO
Objetivo: Identificar a frequência das ações intersetoriais, de inclusão e participação sociais desenvolvidas por Agentes Comunitários de Saúde (ACS). Métodos: Estudo transversal, descritivo e qualitativo, com coleta de dados realizada no Colegiado Gestor Regional (CGR) de Alto Capivari, São Paulo, Brasil. Questionários foram autopreenchidos por 81 Agentes Comunitários de Saúde (ACS), contendo questões sobre as cinco competências que o Ministério da Saúde propõe para os ACS: integração da equipe com a população; planejamento e avaliação; promoção da saúde; prevenção e monitoramento de risco ambiental e sanitário; e prevenção e monitoramento a grupos específicos e morbidades. Resultados: As ações intersetoriais, de inclusão e participação sociais ainda estão em processo de construção no CGR de Alto Capivari, apontando que há dificuldades para sua implementação. As ações de inclusão social são desenvolvidas pelos ACS; já as ações intersetoriais são identificadas e propostas, porém, pouco executadas; e não ocorre participação social dos ACS. Conclusão: A presente pesquisa permitiu identificar que os ACS que trabalham no CGR de Alto Capivari realizam ações de inclusão social e propõem ações intersetoriais, no entanto, essas ações são pouco executadas. Quanto à participação social, os ACS não participam dos Conselhos Locais de Saúde.
\end{abstract}

Descritores: Promoção da Saúde; Estratégia Saúde da Família; Ação Intersetorial; Participação Social.

\section{ABSTRACT}

Objective: To identify the frequency of intersectoral actions, social inclusion and social participation, developed by community health workers (CHWs). Methods: Cross-sectional, descriptive and qualitative study, with data collection performed at the Regional Management Collegiate (RMG) of Alto Capivari, in the state of São Paulo, Brazil. Questionnaires were self-filled by 81 community health workers (CHWs), comprising questions about the five skills proposed by the Ministry of Health for the CHWs: Team integration with the population; planning and evaluation; health promotion; prevention and monitoring of environmental and health hazard; and prevention and monitoring of specific groups and morbidities. Results: Intersectoral actions, social inclusion and participation are still under construction in Alto Capivari RMC, pointing out that there are difficulties in implementing these actions. It is observed that social inclusion initiatives are developed by the CHWs, whereas intersectoral actions are identified and proposed but are poorly implemented; and the social participation of $\mathrm{CHWS}$ does not occur. Conclusion: This research allowed identifying that the CHWS acting in High Capivari RMC carry out social inclusion initiatives and propose intersectoral actions; these actions, however, are poorly executed. As for the social participation, CHWs do not participate in the Local Health Councils.

Descriptors: Health Promotion; Family Health Strategy; Intersectoral Action; Social Participation.

\author{
Lislaine Aparecida Fracolli( ${ }^{(1)}$ \\ Maria Fernanda Pereira \\ Gomes $^{(1)}$ \\ Fabiana Rodrigues Zequini \\ Nabão ${ }^{(2)}$ \\ José Aparecido Alves de \\ Oliveira $^{(2)}$ \\ Cássia Regina Saade Pacheco ${ }^{(2)}$ \\ Tatiane Ferreira Bahia ${ }^{(2)}$
}

1) Universidade de São Paulo - USP - São Paulo (SP) - Brasil

2) Universidade Paulista - UNIP - Assis (SP) - Brasil
Recebido em: 22/12/2014 Revisado em: 19/01/2015 Aceito em: 20/02/2015 


\section{RESUMEN}

Objetivo: Identificar la frecuencia de las acciones intersectoriales, de inclusión y participación social desarrolladas por Agentes Comunitarios de Salud (ACS). Métodos: Estudio transversal, descriptivo y cualitativo en el cual la recogida de datos fue realizada en el Colegiado Gestor Regional (CGR) del Alto Capivari, São Paulo, Brasil. Los cuestionarios fueron contestados por 81 Agentes Comunitarios de Salud (ACS) sobre cuestiones de las cinco competencias que el Ministerio de la Salud ha propuesto para los ACS: la integración del equipo y la población; el planeamiento y la evaluación; la promoción de la salud; la prevención y el control del riesgo ambiental y sanitario; y la prevención y el monitoreo de grupos especificos y morbidades. Resultados: Las acciones intersectoriales, de inclusión y participación sociales aún están en proceso de construcción en el CGR del Alto Capivari señalando que hay dificultades de su implementación. Las acciones de inclusión social son desarrolladas por los ACS mientras las acciones intersectoriales son identificadas y propuestas pero poco ejecutadas y sin participación social de los ACS. Conclusión: Esta investigación permitió identificar que los ACS que trabajan en el CGR del Alto Capivari realizan acciones de inclusión social y proponen acciones intersectoriales, sin embargo, estas acciones son poco ejecutadas. Respecto la participación social, los ACS no participan de los Consejos Locales de Salud.

Descriptores: Promoción de la Salud; Estrategia de Salud Familiar; Acción Intersectorial; Participación Social.

\section{INTRODUÇÃO}

A promoção da saúde vem sendo discutida há mais de 25 anos, com a proposta de reagir ao processo de medicalização da saúde na sociedade e no interior do sistema de saúde ${ }^{(1)}$. Ressalta-se que, dentre as ações que foram incorporadas pela Constituição Brasileira de 1988, a promoção da saúde surge, certamente, como reação à acentuada medicalizaão da saúde na sociedade e no interior do sistema de saúde ${ }^{(1)}$.

Nesse contexto, o Ministério da Saúde propõe para os profissionais que atuam na Atenção Primária à Saúde, de acordo com a Política Nacional de Atenção Básica, a competência "promoção da saúde" como uma atividade elementar no processo de trabalho ${ }^{(2)}$. Nessa conjuntura, a Estratégia Saúde da Família (ESF) foi criada para atender à população - com foco na família, fundamentar sua prática nas necessidades de saúde de cada território e fortalecer os princípios do Sistema Único de Saúde (SUS) no que tange à universalidade do acesso aos serviços de saúde e à integralidade por meio da regulação do acesso aos diversos níveis de assistência à saúde de forma equinânime ${ }^{(2)}$.

Nessa perspectiva, a incorporação do Agente Comunitário de Saúde (ACS) nas Equipes de Saúde da Família pode ser vista como uma tentativa de facilitar o reconhecimento das necessidades de saúde da população, situações de vulnerabilidade social, bem como promover a saúde ${ }^{(3)}$.

A competência "promoção da saúde" atribuída pelo Ministério da Saúde aos ACS compreende várias ações, dentre elas: ação intersetorial: "estabelece parcerias com creches, asilos, escolas, comerciantes, grupos sociais e outros"; ação de inclusão social: "orienta a família e/ ou portador de necessidades especiais quanto às medidas facilitadoras para sua máxima inclusão social"; e ação de participação social: "participa de reuniões do conselho local de saúde ou de outros conselhos locais"(4).

Tomando por base as ações de cunho intersetorial, de inclusão social e de participação social, fica fácil entender o quão importante é o trabalho do ACS na Estratégia Saúde da Família, ao despertar nos grupos sociais o entendimento do empowerment $t^{(5)}$.

Os conceitos de intersetorialidade, inclusão social e participação social partiram dos pressupostos de que a intersetorialidade refere-se à integração dos serviços de saúde e outros órgãos públicos com a finalidade de articular políticas e programas de interesse para a saúde, cuja execução envolva áreas não compreendidas no âmbito do SUS, potencializando, assim, os recursos financeiros, tecnológicos, materiais e humanos disponíveis, evitando duplicidade de meios para fins idênticos ${ }^{(6)}$.

Já a inclusão social pode ser incorporada como um conjunto de meios e ações que combatem a exclusão aos benefícios da vida em sociedade, provocada pela diferença de classe social, origem geográfica, educação, idade, existência de deficiência ou preconceitos raciais. A inclusão visa oferecer aos mais necessitados oportunidades de acesso a bens e serviços, dentro de um sistema que beneficie a todos, e não apenas aos mais $\operatorname{aptos}^{(7)}$. Nesse contexto, a participação popular é considerada o instrumento de governo mais adequado para construir um regime democrático, e o Estado deve "criar um conjunto de mecanismos participativos", visando à incorporação dos cidadãos aos programas de governo local ${ }^{(8)}$.

Para a operacionalização das ações de promoção da saúde, deve-se considerar que o processo saúde-doença é resultante dos determinantes sociais, econômicos, culturais, étnico/raciais, psicológicos e comportamentais, que podem contribuir para o aparecimento das doenças e constituem fatores de risco para a população ${ }^{(9,10)}$. Para tanto, promover a saúde requer articulação entre os diversos setores sociais, além da saúde, garantindo aos usuários o empoderamento para o controle social, inclusão social e emancipação dos sujeitos $^{(10,11)}$.

Embora os temas intersetorialidade, inclusão social e participação social sejam assuntos complexos, busca-se com 
a presente pesquisa mostrar se as ações intersetoriais, de inclusão social e participação social estão sendo realizadas, e qual o envolvimento do ACS na execução dessas ações, visto que esse profissional tem grande influência junto à comunidade em que atua, por suas particularidades socioculturais e poder de capacitar a população para lutar pelos seus direitos na busca de sua qualidade de vida ${ }^{(3)}$.

O objetivo do presente estudo é identificar a frequência das ações intersetoriais, de inclusão social e de participação social desenvolvidas por Agentes Comunitários de Saúde (ACS).

\section{MÉTODOS}

Trata-se de um estudo transversal, descritivo e quantitativo, realizado em 2011. O cenário de estudo escolhido para a pesquisa, o Colegiado de Gestão Regional (CGR) de Alto Capivari, São Paulo, Brasil, é pertencente à Rede Regional de Atenção à Saúde (RRAS) 11, de Presidente Prudente. Esse CGR é composto pelos municípios de Iepê (7.628 mil habitantes), João Ramalho (4.180 mil habitantes), Nantes (2.750 mil habitantes), Quatá (12.909 mil habitantes) e Rancharia (28.809 mil habitantes).

Dos 97 Agentes Comunitários de Saúde que trabalhavam nas Equipes de Saúde da Família do CGR de Alto Capivari, em janeiro de 2011, 81 aceitaram participar da pesquisa.

Realizou-se a coleta de dados por meio da aplicação de um questionário com questões tipo Likert ${ }^{(12)}$ a partir das cinco competências que o Ministério da Saúde propõe para os ACS, sendo elas: integração da equipe com a população; planejamento e avaliação; promoção da saúde; prevenção e monitoramento de risco ambiental e sanitário; e prevenção e monitoramento a grupos específicos e morbidades ${ }^{(4)}$.

No presente estudo, pretendeu-se analisar em particular seis facetas que compõem a competência "promoção da saúde": A1 - Propõe ações que utilizam as diversas secretarias existentes do município; A2 - Executa ações que atuam em parceria com outras secretarias existentes no município; A3 - Estabelece parcerias com creches, asilos, escolas, comerciantes, grupos sociais e outros; A4 - Orienta a família e/ou portador de necessidades especiais quanto às medidas facilitadoras para sua máxima inclusão social; A5 - Apoia ações sociais de alfabetização de crianças, adolescentes, jovens e adultos; A6 - Participa de reuniões do conselho local de saúde ou de outros conselhos.

Os pesquisadores disponibilizaram, em visita agendada na unidade de saúde, os questionários aos Agentes Comunitários de Saúde que aceitaram participar da pesquisa. Nesse caso, os questionários foram autopreenchidos numa média de 20 minutos pelos agentes e recolhidos pelos pesquisadores.
Os dados foram organizados e analisados segundo a frequência simples das ocorrências, com auxílio do SPSS.

A presente pesquisa obteve a aprovação do Comitê de Ética em Pesquisa da Escola de Enfermagem da Universidade de São Paulo, sob o parecer n. 963/2010/ CEP-EEUSP, e pelos Secretários Municipais de Saúde dos cinco municípios.

\section{RESULTADOS}

No município de Iepê, observou-se que a ação intersetorial A1 tem maior frequência de realização mensalmente; já a ação intersetorial A2 é mais realizada diariamente; enquanto a ação A3 é mais realizada mensalmente (Tabela I).

Outro ponto importante a destacar é que, no município de Iepê, a ação A1 possui o percentual de 53,8\% de nunca realizada, e a ação A2 possui o percentual de $46,1 \%$ de nunca realizada (Tabela I).

Quanto às ações intersetoriais no município de João Ramalho, em relação à ação A1, observou-se padrão de maior realização diariamente; a ação A2 apresentou maior realização diariamente e mensalmente, enquanto a ação A3 é mais realizada mensalmente. No município de João Ramalho, as ações intersetoriais A1 e A3 são realizadas raramente no percentual de $40 \%$ e $50 \%$, e A2 possui percentual igual a 50\% de nunca realizada (Tabela I).

Em Nantes, as ações A1, A2 e A3 são mais realizadas mensalmente. Destaca-se que as ações A1 e A2 são realizadas raramente sob o percentual de $80 \%$ e $60 \%$. No município de Quatá, observa-se que as ações A1, A2 e A3 são pouco realizadas e apresentam os respectivos percentuais de realização raramente de 73,7\%, 84,2\% e 78,9\% (Tabela I).

O município de Rancharia apresentou maior frequência de realização das ações A1, A2 e A3 diariamente, observando-se que as ações A1, A2 e A3 são realizadas raramente sob o respectivo percentual de $32,3 \%, 52,9 \%$ e $35,3 \%$ (Tabela I).

A Tabela I mostra que, no município de Iepê, a ação A4 é realizada todos os dias sob o percentual de $46,1 \%$ e raramente realizada também sob o percentual de $46,1 \%$; a ação A5 é pouco realizada, apresentando o percentual $61,5 \%$ de nunca realizada. Em João Ramalho, as ações de inclusão social, A4 e A5, possuem frequência de realização significativa de $80 \%$.

No município de Nantes, os ACS responderam que realizam as ações A4 diariamente e A5 mensalmente. Com relação à ação A4, destaca-se o percentual de $60 \%$ de raramente realizada. Em Quatá, destaca-se que A5 possui $57,8 \%$ de realização raramente. No município de Rancharia, 
os ACS participantes da presente pesquisa responderam que realizam as ações A4 e A5 diariamente (Tabela I).

A ação A6, de acordo com a Tabela I, é pouca desenvolvida nos municípios do CGR de Alto Capivari, destacando-se com melhor percentual de realização o município de Nantes, com $20 \%$ de realização diariamente. Os municípios de João Ramalho e Quatá apresentaram 80\% e $73,68 \%$, respectivamente, de realização raramente, o que indica que os ACS têm pouca atividade no controle social do SUS.

Analisando as frequências mensais de participação social dos ACS em seus respectivos municípios, encontrou- se que, em Iepê, 7,7\% dos ACS responderam que participam dos Conselhos Locais de Saúde (CLS); em João Ramalho, $10 \%$ dos ACS referem participar do CLS; já em Nantes, $20 \%$ dos ACS dizem participar; $30 \%$ dos ACS de Rancharia referem participar mensalmente, enquanto os ACS de Quatá referem que participam mensalmente dos CLS. Nesse contexto, verifica-se que os ACS pouco participam dos CLS, órgãos cuja finalidade é garantir a participação dos usuários e funcionários, juntamente com a administração, na gestão da saúde e controle das ações e serviços da Unidade de Saúde (Tabela I).

Tabela I - Frequência das ações intersetoriais, de inclusão social e participação social de acordo com os agentes comunitários de saúde. Colegiado Gestor Regional de Alto Capivari-SP, 2011.

\begin{tabular}{|c|c|c|c|c|c|c|}
\hline \multicolumn{7}{|l|}{ IEPÊ } \\
\hline Ações n(\%) & A1 & A2 & A3 & A4 & A5 & A6 \\
\hline Todos os dias & $1(7,7)$ & $4(30,8)$ & $2(15,4)$ & $6(46,1)$ & $3(23,1)$ & $0(0)$ \\
\hline Todos as semanas & $1(7,7)$ & $0(0)$ & $0(0)$ & $0(0)$ & $1(7,7)$ & $1(7,7)$ \\
\hline Todos os meses & $4(30,8)$ & $1(7,7)$ & $3(23,1)$ & $1(7,7)$ & $0(0)$ & $1(7,7)$ \\
\hline Raramente & $0(0)$ & $2(15,4)$ & $5(38,5)$ & $6(46,1)$ & $1(7,7)$ & $1(7,7)$ \\
\hline Nunca & $7(53,8)$ & $6(46,1)$ & $3(23,1)$ & $0(0)$ & $8(61,5)$ & $10(76,9)$ \\
\hline \multicolumn{7}{|l|}{ JOÃO RAMALHO } \\
\hline Ações n(\%) & A1 & $\mathbf{A} 2$ & A3 & A4 & A5 & A6 \\
\hline Todos os dias & $2(20)$ & $2(20)$ & $1(10)$ & $8(80)$ & $8(80)$ & $0(0)$ \\
\hline Todas as semanas & $1(10)$ & $0(0)$ & $0(0)$ & $1(10)$ & $0(0)$ & $0(0)$ \\
\hline Todos os meses & $1(10)$ & $2(20)$ & $3(30)$ & $1(10)$ & $1(10)$ & $1(10)$ \\
\hline Raramente & $4(40)$ & $1(10)$ & $5(50)$ & $0(0)$ & $1(10)$ & $8(80)$ \\
\hline Nunca & $0(0)$ & $5(50)$ & $1(10)$ & $0(0)$ & $0(0)$ & $1(10)$ \\
\hline \multicolumn{7}{|l|}{ NANTES } \\
\hline Ações n(\%) & A1 & $\mathbf{A 2}$ & A3 & A4 & A5 & A6 \\
\hline Todos os dias & $0(0)$ & $0(0)$ & $1(20)$ & $1(20)$ & $1(20)$ & $1(20)$ \\
\hline Todas as semanas & $0(0)$ & $0(0)$ & $0(0)$ & $0(0)$ & $0(0)$ & $0(0)$ \\
\hline Todos os meses & $1(20)$ & $1(20)$ & $3(60)$ & $1(20)$ & $2(40)$ & $1(20)$ \\
\hline Raramente & $4(80)$ & $3(60)$ & $0(0)$ & $3(60)$ & $2(40)$ & $2(40)$ \\
\hline Nunca & $0(0)$ & $1(20)$ & $1(10)$ & $0(0)$ & $0(0)$ & $0(0)$ \\
\hline \multicolumn{7}{|l|}{ QUATÁ } \\
\hline Ações n(\%) & A1 & A2 & A3 & A4 & A5 & A6 \\
\hline Todos os dias & $1(5,3)$ & $0(0)$ & $0(0)$ & $4(21)$ & $3(15,8)$ & $0(0)$ \\
\hline Todas as semanas & $3(15,8)$ & $1(5,3)$ & $0(0)$ & $1(5,3)$ & $0(0)$ & $1(5,3)$ \\
\hline Todos os meses & $0(0)$ & $1(5,3)$ & $0(0)$ & $8(42,1)$ & $5(26,3)$ & $0(0)$ \\
\hline Raramente & $14(73,7)$ & $16(84,2)$ & $15(78,9)$ & $5(23,3)$ & $11(57,9)$ & $14(73,7)$ \\
\hline Nunca & $1(5,3)$ & $1(5,3)$ & $12(35,3)$ & $1(5,3)$ & $0(0)$ & $4(21)$ \\
\hline \multicolumn{7}{|l|}{ RANCHARIA } \\
\hline Ações n(\%) & A1 & A2 & A3 & A4 & A5 & A6 \\
\hline Todos os dias & $16(47)$ & $6(17,6)$ & $7(20,6)$ & $22(64,7)$ & $21(61,8)$ & $1(2,9)$ \\
\hline Todas as semanas & $3(8,8)$ & $2(8,8)$ & $4(11,8)$ & $3(8,8)$ & $1(2,9)$ & $0(0)$ \\
\hline Todos os meses & $2(5,9)$ & $3(8,8)$ & $10(29,4)$ & $5(14,7)$ & $2(5,9)$ & $10(29,4)$ \\
\hline Raramente & $11(32,3)$ & $18(52,9)$ & $12(35,3)$ & $4(11,8)$ & $9(26,5)$ & $19(55,9)$ \\
\hline Nunca & $2(5,9)$ & $2(5,9)$ & $1(2,9)$ & $0(0)$ & $1(2,9)$ & $4(11,8)$ \\
\hline
\end{tabular}

A1-Propõe ações que utilizam as diversas secretarias existentes do município; A2-Executa ações que atuam em parceria com outras secretarias existentes no município; A3-Estabelece parcerias com creches, asilos, escolas, comerciantes, grupos sociais e outros; A4-Orienta à família e/ou portador de necessidades especiais quanto às medidas facilitadoras para sua máxima inclusão social; A5-Apoia ações sociais de alfabetização de crianças, adolescentes, jovens e adultos; A6-Participa de reuniões do conselho local de saúde ou de outros conselhos. 


\section{DISCUSSÃO}

Para a resolução de problemas complexos, muitas vezes, é imprescindível a realização de ações intersetoriais e olhar a saúde a partir de seus determinantes e condicionantes ${ }^{(3)}$. Nesse contexto, a ESF, por estar mais próxima da população e conhecer suas necessidades e vulnerabilidades, pode trabalhar melhor o estabelecimento de parcerias com outros setores, no entanto, a operacionalização de ações intersetoriais não é favorecida pelas políticas que regem os vários setores sociais, devido ao engessamento e à falta de pontos de congruência que facilitem o desenvolvimento dessas ações ${ }^{(3)}$.

A intersetorialidade é uma estratégia em construção, da qual os diferentes atores, setores e segmentos sociais ainda estão se apropriando ${ }^{(13)}$. Essa informação corrobora com um estudo realizado no município do Rio de Janeiro, o qual identificou que a maior preocupação dos ACS era revelar o compromisso do sistema de saúde com a comunidade ${ }^{(13)}$. Os ACS, contudo, percebiam-se impossibilitados de promover a saúde dos indivíduos e das comunidades diante da necessidade de medidas intersetoriais para a reversão da miséria e de outros determinantes importantes do processo saúde-doença ${ }^{(13)}$. Dessa forma, na presente pesquisa, verificou-se que as ações intersetoriais são propostas pelos ACS que trabalham no CGR de Alto Capivari, no entanto, quanto à execução dessas ações, verificou-se que são pouco realizadas. Os agentes comunitários, ao realizarem as visitas domiciliares, deparam-se com problemas que extrapolam o setor de saúde. Após a identificação dos problemas, passam as informações para a equipe de saúde da qual fazem parte; a equipe de saúde, por sua vez, por despreparo, falta de capacitação e dificuldades presentes na articulação da saúde com outros setores, não resolve os problemas e não melhora a situação de saúde da população.

Situações que demandam a intersetorialidade, para serem resolvidas, muitas vezes são deixadas de lado pelos profissionais de saúde, por acharem que estão impossibilitados a desenvolver ações intersetoriais, aplicando uma abordagem baseada principalmente no controle de doenças crônicas e na prevenção de outros $\operatorname{agravos}^{(3)}$.

Frequentemente, os profissionais de saúde sentemse impotentes diante dos determinantes sociais da saúde, deparando-se com problemas como a miséria, o desemprego, a falta de higiene e a fome, dentre tantas outras situações para as quais não há cura imediata, mas são tão graves que precisam ser cuidadas ${ }^{(13)}$.

Para o desenvolvimento de uma cultura de trabalho intersetorial, a ESF necessita, entre outras coisas, conhecer os condicionantes da qualidade de vida da população, promover ações integradas e parcerias com diferentes organizações para o enfrentamento conjunto dos problemas identificados ${ }^{(14)}$.

Nesse sentido, a capacitação e a educação permanente da equipe multiprofissional, em especial do ACS, são fundamentais para que os profissionais busquem atender às necessidades das famílias, direcionando o olhar e as ações de saúde para além das práticas curativas ${ }^{(3)}$. É necessário, ainda, que os serviços de saúde e seus profissionais adotem uma forma de interação inovadora, contextualizada e em consonância com os valores das famílias nos cenários social e político ${ }^{(15)}$.

Embora existam muitos desafios para a operacionalização e construção da intersetorialidade, a implantação de políticas públicas intersetoriais para resolução de problemas encontrados nas comunidades deve ser uma meta e prioridade dos gestores de saúde das três esferas de governo ${ }^{(3)}$.

No presente estudo, as ações intersetoriais são pouco realizadas pelos ACS, bem como pelos outros profissionais que atuam na saúde de uma maneira geral ${ }^{(16)}$. Para tanto, a implantação da intersetorialidade requer decisão política, pois implica em mudanças na organização das estruturas de poder e requer, também, mudança na lógica de governo, de organização do trabalho, para a prevenção ou solução de problemas existentes em um território geográfico ${ }^{(16)}$.

Na presente pesquisa, foi possível observar que os ACS que trabalham na ESF do CGR de Alto Capivari realizam ações de inclusão social. Dessa forma, o conhecimento das necessidades de saúde da população por parte desses profissionais permite que as informações possam ser difundidas às famílias e incorporadas na prática.

Hoje, no Brasil, milhares de pessoas com algum tipo de deficiência estão sendo discriminadas nas comunidades em que vivem ou sendo excluídas do mercado de trabalho ${ }^{(17)}$. O processo de exclusão social de pessoas com deficiência ou alguma necessidade especial é tão antigo quanto a socialização do homem ${ }^{(17)}$. Cada deficiência acaba acarretando em um tipo de comportamento e suscitando diferentes formas de reações, preconceitos e inquietações ${ }^{(17)}$. O quadro fica mais sério, tendo em vista que a tendência dos profissionais da saúde é sempre ressaltar, no diagnóstico, os aspectos limitantes da deficiência, pois, invariavelmente, são eles que primeiro são chamados para dar o diagnóstico conclusivo ${ }^{(17)}$. Os médicos raramente esclarecem ou informam aos familiares de portadores de deficiência as possibilidades de desenvolvimento, as formas de superação das dificuldades, os locais de orientação familiar, os recursos de estimulação precoce, os centros de educação e de terapia ${ }^{(17)}$. A esses familiares, pede-se que aceitem uma realidade que não desejam e que não é prevista, uma realidade que os meios sociais e a mídia pouco abordam, e quando o fazem é de maneira superficial, 
às vezes preconceituosa e sem apresentar os caminhos para a inclusão social ${ }^{(17)}$.

Tomando por base as ações desenvolvidas pelos ACS, é muito importante que eles realizem ações que promovam a inclusão social por meio da capacitação da população no conhecimento de seus direitos e o esclarecimento de dúvidas relativas às atividades de inclusão social disponíveis nos municípios $^{(3)}$.

A participação social dos ACS nos espaços políticos de decisão sobre a política de saúde regional tem sido escassa, conforme mostram os resultados da presente pesquisa. Os ACS são profissionais que podem estimular a participação e o controle social, contudo, fica difícil imaginar como eles estimulam essa participação sem estarem envolvidos no processo $^{(3)}$. Sabe-se que os Conselhos Locais de Saúde são instâncias para o planejamento, a execução e a avaliação das ações, não só no âmbito local, mas nos âmbitos municipal, estadual e federal ${ }^{(3)}$.

Nessa perspectiva, o controle social se constitui como importante ferramenta para a melhoria da situação de saúde da população, pois é um espaço político que, pela proximidade e vivência da realidade de sua região adscrita, lida com as necessidades, os interesses, as dificuldades e os anseios da comunidade ${ }^{(18,19)}$. Assim, pode ser um propulsor de mudanças diretamente relacionado com a promoção da saúde, entendendo a participação popular como fundamental na eleição de prioridades, nas decisões e na elaboração de estratégias para alcançar o melhor nível de saúde, desenvolvendo políticas que partam dos problemas e necessidades de saúde ${ }^{(18,19)}$.

O ACS deve desenvolver sua habilidade política para estimular o controle social da comunidade em que atua e desenvolver a autonomia e o empowerment ${ }^{(20)}$. A habilidade política do ACS confere a esse profissional grande autonomia nas ações relativas à resolução de problemas de saúde da população ${ }^{(20)}$.

Nesse aspecto, o ACS tem um papel importante, pois, mesmo que não participe dos conselhos como membro, deveria participar das reuniões mensais para comunicar à população as informações discutidas nesses espaços, afinal, ele é o profissional da ESF que está cotidianamente com a população ${ }^{(22)}$.

Nessa perspectiva, a promoção da saúde exige a mobilização de recursos políticos, humanos e financeiros que extrapolam o âmbito da saúde, colocando a intersetorialidade como um desafio para implantar práticas de promoção da saúde ${ }^{(21)}$. Outro ponto importante é que a participação social é considerada um dos pilares para a promoção da saúde, cujo objetivo é a autonomia e a emancipação dos usuários, para a sua máxima inclusão social ${ }^{(14)}$.
A presente pesquisa contribui para retratar a frequência de realização das ações intersetoriais, de inclusão e participação sociais, apresentando a limitação de não descrever como essas ações são realizadas na prática. Outra fragilidade desta pesquisa é que ela engloba apenas a participação do ACS, excluindo os outros membros da equipe de ESF. Tendo em vista os aspectos observados, é importante que haja o incentivo ao desenvolvimento das ações intersetoriais, de inclusão e participação sociais, para o fortalecimento da promoção da saúde. Dessa forma, esforços são necessários na busca da reorganização política e setorial, a fim de facilitar a execução das ações intersetoriais e de inclusão social pelos profissionais de saúde. Portanto, a participação social deve ser uma cultura vivida e difundida pelos profissionais e gestores de saúde para o empoderamento dos usuários do sistema de saúde.

\section{CONCLUSÃO}

A presente pesquisa permitiu identificar que os ACS que trabalham no CGR de Alto Capivari realizam ações de inclusão social e propõem ações intersetoriais, no entanto, elas ações são pouco executadas. Quanto à participação social, os ACS não participam dos Conselhos Locais de Saúde.

\section{REFERÊNCIAS}

1. Czerenia DF, Freitas CM. Promoção da saúde: conceitos, reflexões, tendências. Rio de Janeiro: FIOCRUZ; 2003.

2. Ministério da Saúde (BR), Secretaria de Atenção à Saúde, Departamento de Atenção Básica. Política Nacional de Atenção Básica. Brasília: Ministério da Saúde; 2012.

3. Fracolli LA, Gomes MFPG, Pinheiro DGM, Castro DFA. Avaliação das ações de promoção da saúde desenvolvidas pelos agentes comunitários de saúde. Mundo Saúde. 2013;37(4):411-8.

4. Ministério da Saúde (BR), Ministério da Educação. Referencial curricular para o curso técnico de agente comunitário de saúde: área profissional. Brasília: Ministério da Saúde; 2004.

5. Pinto AAM, Fracolli LA. O trabalho do agente comunitário de saúde na perspectiva da promoção da saúde: considerações práxicas. Rev Eletrônica Enferm [periódico na Internet]. 2010; [acesso em 2014 Mar 12];12(4):766-9. Disponível em: http://www.revistas. ufg.br/index.php/fen/article/view/7270/8501. 
6. Associação Paulista de Medicina - APM. SUS: o que você precisa saber sobre o Sistema Único de Saúde. São Paulo: Atheneu; 2004.

7. Stainback W, Stainback S. Inclusão: um guia para educadores. Porto Alegre: Artmed; 1999.

8. Valla VV, Stotz EM. Participação popular, educação, saúde teoria e prática. Rio de Janeiro: Relume-dumará; 1993.

9. Costa BVL, Mendonça RD, Santos LC, Peixoto SV, Alves M, Lopes ACS. Academia da Cidade: um serviço de promoção da saúde na rede assistencial do Sistema Único de Saúde. Ciênc Saúde Coletiva. 2013;18(1):95102.

10. Santos DS, Tenório EA, Brêda MZ, Mishima SM. Processo saúde/doença e estratégia saúde da família: o olhar do usuário. Rev Latinoam Enferm. 2014;22(6):918-25.

11. Bydlowski CR, Lefevre AMC, Pereira IMTB. Promoção da saúde e a formação cidadã: a percepção do professor sobre cidadania. Ciênc Saúde Coletiva. 2011;16(3):1771-80.

12. Santos LPGS, Fracolli LA. O Agente Comunitário de Saúde: possibilidades e limites para a promoção da saúde. Rev Esc Enferm USP. 2010;44(1):76-83.

13. Barbosa VBA, Ferreira MLSM, Barbosa PMK. Educação permanente em saúde: uma estratégia para a formação dos agentes comunitários de saúde. Rev Gaúcha Enferm. 2012;33(1):56-63.

14. Pereira IC, Oliveira MAC. O trabalho do agente comunitário na promoção da saúde: revisão integrativa da literatura. Rev Bras Enferm. 2013;66(3):412-9.

15. Noronha MGRCS, Cardoso OS, Moraes TNP, Centa ML. Resiliência: nova perspectiva na Promoção da Saúde da Família?. Ciênc Saúde Coletiva. 2009;14(2):497-506.

16. Warschauer M, Carvalho YM. O conceito "Intersetorialidade": contribuições ao debate a partir do Programa Lazer e Saúde da Prefeitura de Santo André/SP. Saúde Soc. 2015;23(1):191-203.
17. Maciel MRC. Portadores de deficiência: a questão da inclusão social. São Paulo Perspec. [periódico na internet]. 2000 jun. [acesso em 2014 Mar 16];14(2):51-6. Disponível em: http:// www.scielo.br/scielo.php?script=sci_arttext\&pid $=$ S0102-88392000000200008.

18. Nogueira FCP, Lima LHO, Wolfovitch RGS, Nascimento SM, Lopes SRM. Implantação de um conselho local de saúde: desafios da prática do controle social. Rev Baiana Saúde Pública. 2008;32(1):104-10.

19. Staliano P, Araujo TCCF. Promoção da Saúde e Prevenção de Doenças: Um Estudo com Agentes Comunitários de Saúde. Rev Psicologia Saúde. 2011;3(1):43-51.

20. Nunes MO, Trad LB, Almeida BA, Homem CR, Melo MCIC. O agente comunitário de saúde: construção da identidade desse personagem híbrido e polifônico. Cad Saúde Pública. 2002;18(6):1639-46.

21. Silva KL, Sena RR, Lima KMSV, Martins BR, Santos JBO. Promoção da saúde e intersetorialidade em um município da região metropolitana de Belo Horizonte/ Minas Gerais. Rev APS. 2013;16(2):165-72.

22. Silva ERP, Cazola LHO, Cheade MFM, Pícoli RP. Atuação dos agentes comunitários de saúde na estratégia saúde da família. Cogitare Enferm. 2012;17(4):635-41.

\section{Endereço do primeiro autor:}

Lislaine Aparecida Fracolli

Escola de Enfermagem da Universidade de São Paulo EEUSP

Av. Dr. Enéas de Carvalho Aguiar, 419

Bairro: Cerqueira César

CEP: 05403-000 - São Paulo - SP - Brasil

E-mail: lislaine@usp.br

\section{Endereço para correspondência:}

Maria Fernanda Pereira Gomes

Escola de Enfermagem da Universidade de São Paulo EEUSP

Av. Dr. Enéas de Carvalho Aguiar, 419

Bairro: Cerqueira César

CEP: 05403-000 - São Paulo - SP - Brasil

E-mail: mferpg@usp.br 